\title{
REFLECTION AND TRANSMISSION OF SEISMIC WAVES UNDER INITIAL STRESS AT THE EARTH'S CORE-MANTLE BOUNDARY
}

\author{
SUKHENDU DEY and SUSHIL KUMAR ADDY \\ Department of Physics and Mathematics \\ Indian School of Mines \\ Dhanbad - 826004 \\ INDIA
}

(Received December 19, 1978)

ABSTRACT. In the present paper the influence of the initial stress is shown on the reflection and transmission of $P$ waves at the core-mantle boundary. Taking a particular value of the inherent initial stress, the variations of reflection and transmission coefficients with respect to the angle of emergence are represented by graphs. These graphs when compared with those having no initial stress show that the effect of the initial stress is to produce a reflected $P$ and $S$ waves with numerically higher amplitudes but a transmitted $P$ wave with smaller amplitude. A method is also indicated in this paper to calculate the actual value of the initial stress near the core-mantle boundary by measuring the amplitudes of incident and reflected $P$ waves.

KEY WORDS AND PHRASES. Seismic Waves, Reflection and Transmission of $P$ Waves. 1980 MATHEMATICS SUBJECT CLASSIFICATION CODES. $73020,73 N 10$ 


\section{INTRODUCTION.}

The reflection and transmission of seismic waves at the earth's core-mantle boundary have been discussed by Dana [1], Ibrahim [2] and many other investigators. From their discussions we see that the reflected and transmitted waves are dependent on elastic parameters, densities and the angle of incidence. But the mantle and core contain a considerable amount of initial stress which is compressive and supposed to be hydrostatic in nature (Jeffreys, [3]). The present paper shows that this initial stress has also a significant effect on the reflected and transmitted waves at the core-mantle boundary. The paper is constructed on the assumption that the core is liquid and that there is no discontinuity of initial stress at the core-mantle boundary. For simplicity, only $P$ wave incident from the mantle side has been considered. This $P$ wave produces reflected $P(P C P)$, reflected $S$ (PCS) and transmitted $P$ waves, each of which is influenced by the initial stress. Taking a particular value of the inherent initial stress, the numerical values of reflection and transmission coefficients for different angles of emergence have been calculated and the results are given by graphs. The corresponding graphs when the initial stress is not considered are also given for comparison. From these graphs it is found that the initial stress increases the numerical values of the coefficients for the reflected $P$ and $S$ waves but decreases the same for the transmitted $P$ waves.

It is shown at last that from the expression of the ratio of the coefficients for the reflected and incident $P$ waves we may calculate the actual value of the initial stress near the core-mantle boundary.

2. FORMULATION AND SOLUTION OF THE PROBLEM.

Let us assume that $y=0$ be the boundary of the earth's core (Fig. 1). The mantle and core are supposed to be homogeneous and isotropic elastic media. Let $\mathrm{H}$ be the initial compressive hydrostatic stress just outside and inside the core 
including the boundary.

The wave equations with initial hydrostatic stress are the same as those without initial stress (Dey, [4]). They are given by

$$
\begin{aligned}
& \nabla_{\phi}^{2}=\frac{\rho}{(\lambda+2 \mu)} \frac{\partial^{2} \phi}{\partial t^{2}} \\
& \nabla^{2} \psi=\frac{\rho}{\mu} \frac{\partial^{2} \psi}{\partial t^{2}}
\end{aligned}
$$

where $\rho$ is the density, $\lambda$ and $\mu$ are Lame's constants and $\nabla^{2}=\frac{\partial^{2}}{\partial x^{2}}+\frac{\partial^{2}}{\partial y^{2}}$.

We shall consider only $P$ wave incident from the mantle side. The solutions of equations (2.1ab) are

$$
\left.\begin{array}{l}
\phi=A \exp [i k(c t-x+a y)]+A_{1} \exp [i k(c t-x-a y)] \\
\psi=B_{1} \exp [i k(c t-x-b y)],
\end{array}\right\}
$$

where $k$ is the wave number, $a$ is connected with the angle of emergence $e$ by the relation $\mathrm{a}=\tan \mathrm{e}$, and

$$
\begin{array}{ll}
a=\sqrt{c^{2} / \alpha^{2}-1}, & b=\sqrt{c^{2} / \beta^{2}-1}, \\
\alpha=\sqrt{\frac{\lambda+2 \mu}{\rho}}, \text { and } \quad \beta=\sqrt{\mu / \rho} .
\end{array}
$$

For the outer core, which is supposed to be a liquid, the transmitted $P$ wave is given by

$$
\phi^{\prime}=A^{\prime} \exp i k\left[\left(c t-x+a^{\prime} y\right)\right],
$$

where $a^{\prime}=\sqrt{c^{2} / \alpha^{-2}-1}, \alpha^{-}=\sqrt{\lambda^{\prime} / \rho^{-}}, \lambda^{-}$is the Lame constant and $\rho^{\prime}$ is the density just inside the core.

The boundary conditions require that the vertical displacement $\mathrm{v}$ and the incremental boundary force $\Delta f_{y}$ per unit initial area are continuous across the 
surface $y=0$ and the incremental tangential force $\Delta f_{x}$ per unit initial area vanishes at the same surface. These conditions are equivalent to

$$
v=v^{\prime}, \Delta f_{x}=0, \Delta f_{y}=\Delta f_{y}^{\prime} \text { at } y=0
$$

The quantities without and with primes refer to the mantle and core sides respectively.

We write the displacements $u, v$ in terms of the functions $\phi$, by the relations

$$
u=\frac{\partial \phi}{\partial x}-\frac{\partial \psi}{\partial y}, \quad v=\frac{\partial \phi}{\partial y}+\frac{\partial \psi}{\partial x}
$$

$\Delta f_{x}$ and $\Delta f_{y}$ are given in Biot [5]

$$
\Delta f_{x}=s_{12}+H \frac{\partial v}{\partial x}, \Delta f_{y}=s_{22}-H \frac{\partial u}{\partial x},
$$

where $s_{i j}$ are incremental stresses and are expressed by (Biot, [5])

$$
s_{12}=2 \mu e_{x y}, s_{22}=s+2 \mu e_{y y}
$$

when the initial stress is hydrostatic.

Equations (2.5), (2.6) and (2.7) change the boundary conditions to

$$
\begin{aligned}
& \frac{A_{1}}{A}+\frac{1}{a} \frac{B_{1}}{A}+\frac{a^{\prime}}{a} \frac{A^{\prime}}{A}=1 \\
& \frac{A_{1}}{A}-\frac{\mu\left(b^{2}-1\right)-H}{(2 \mu+H) a} \frac{B_{1}}{A}=1 \\
& \frac{A_{1}}{A}+\frac{(2 \mu+H) b}{\mu\left(b^{2}-1\right)} \frac{B_{1}}{A}-\frac{\delta \mu\left(b^{2}+1\right)-H}{\mu\left(b^{2}-1\right)-H} \frac{A^{\prime}}{A}=-1,
\end{aligned}
$$

where $\delta=\rho \% \rho$.

Solving equations $(2.8)$ we obtain

$$
\frac{A_{1}}{A}=\frac{M-N}{M+N}, \frac{B_{1}}{A}=\frac{P}{M+N}, \frac{A^{\prime}}{A}=\frac{Q}{M+N},
$$


where $\quad M=(2 \mu+H)^{2} \quad b a^{\prime} a+\left[\delta \mu\left(b^{2}+1\right)-H\right] \mu\left(b^{2}+1\right) a$,

$$
\begin{aligned}
& N=\left[\mu\left(b^{2}-1\right)-H\right]^{2} a^{\prime}, \\
& P=-2(2 \mu+H)\left[\mu\left(b^{2}-1\right)-H\right] a^{\prime} a,
\end{aligned}
$$

and

$$
Q=2 \mu\left[\mu\left(b^{2}-1\right)-H\right]\left(b^{2}+1\right) a .
$$

Introducing the non-dimensional parameters $p=\alpha / \beta, q=\alpha / \alpha^{\prime}$ and $\zeta=H / 2 \mu$ to the relations (2.9) we obtain

$$
\frac{A_{1}}{A}=\frac{M_{0}-N_{0}}{M_{0}+N_{0}}, \frac{B_{1}}{A}=\frac{P_{0}}{M_{0}+N_{0}}, \frac{A^{\prime}}{A}=\frac{Q_{0}}{M_{0}+N_{0}},
$$

where

$$
\begin{aligned}
M_{0} & =4(1+\zeta)^{2}\left(p^{2} \sec ^{2} e-1\right)^{1 / 2}\left(q^{2} \sec ^{2} e-1\right)^{1 / 2} \tan e \\
& +\delta\left(q^{2} \sec ^{2} e-2 \zeta \theta\right)\left(p^{2} / q^{2}\right) p^{2} \sec ^{2} e \tan e \\
N_{0} & =\left(p^{2} \sec ^{2} e-2-2 \zeta\right)^{2}\left(q^{2} \sec ^{2} e-1\right)^{1 / 2} \\
P_{0} & =-4(1+5)\left(p^{2} \sec ^{2} e-2-2 \zeta\right)\left(q^{2} \sec ^{2} e-1\right)^{1 / 2} \tan e
\end{aligned}
$$

and

$$
Q_{0}=2 p^{2} \sec ^{2} e\left(p^{2} \sec ^{2} e-2-2 \zeta\right) \text { tan e with } \theta=\mu / \lambda^{\prime} \text {. }
$$

From (2.10) it is clear that $A_{1} / A, B_{1} / A$ and $A-/ A$ depend on $\zeta$ in addition to $\theta$, e and elastic parameters.

\section{NUMERICAL RESULTS AND CONCLUSIONS}

Following Bullen $[6]$ we have assumed that $p=1.89, q=1.71, \delta=1.71$, $\theta=0.50$ and $\zeta=0.22$. Taking these values of $p, q, \delta, \theta$ and $\zeta$, the numerical values of $A_{1} / A, B_{1} / A$ and $A \% / A$ have been calculated for different values of $e$ between $0^{\circ}$ to $90^{\circ}$ at the interval of $10^{\circ}$. The results are given graphically in Fig. 2. The corresponding graphs when 5 vanishes are also shown in the same figure. From these graphs we infer the following things: 


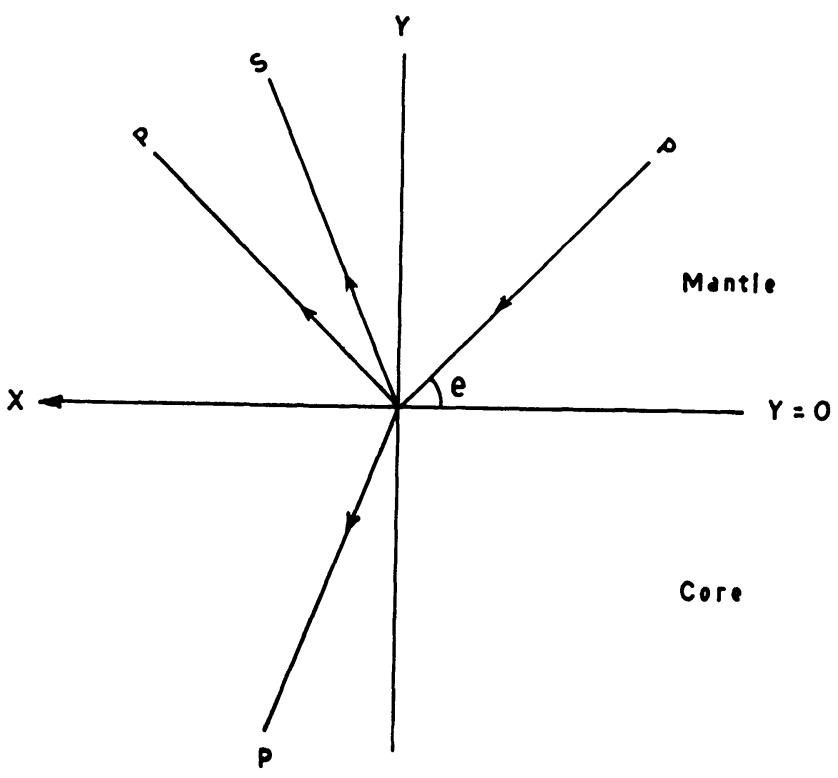

P WAVE INCIDENT AT THE CORE-MANTLE BOUNDARY

FIG.I.

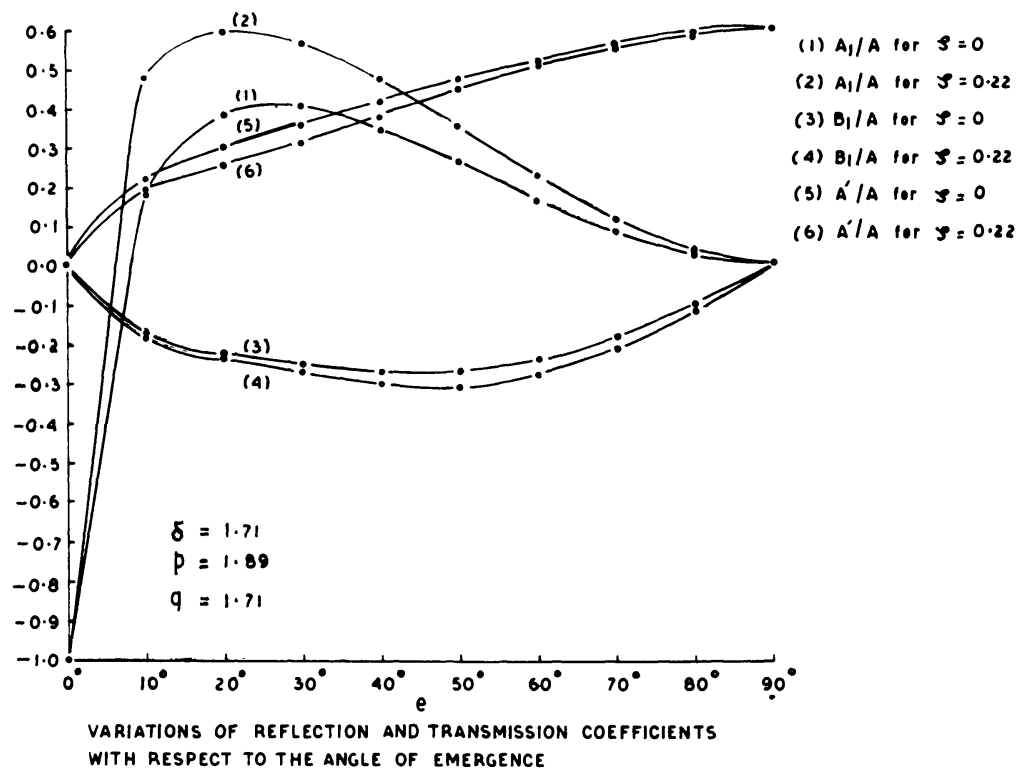

FIG. 2. 
At the grazing and normal incidences, i.e. when $e=0^{\circ}$ and $90^{\circ}$ respectively, $A_{1} / A, B_{1} / A$ and $A^{\prime} / A$ are independent of 5 . When $e=0^{\circ}$, we get $A_{1} / A=-1$ and $B_{1} / A=A^{\prime} / A=0$. This indicates that there is a total reflection with reversal of phase at the grazing incidence. When $e=90^{\circ}$, we get $A_{1} / A=B_{1} / A=0$ and $A^{\prime} / A=0.58$. This means that no reflection of $P$ wave occurs but a part of it is transmitted through the core-mantle boundary at the normal incidence. When e lies between $0^{\circ}$ and $90^{\circ}, 5$ increases the numerical of $A_{1} / A$ and $B_{1} / A$ but decreases $A^{\prime} / A$. When $\zeta$ is not taken into account, $A_{1} / A$ attains the maximum value 0.4 near $\mathrm{e}=25^{\circ}$. By the consideration of $\zeta$, the maximum value of $\mathrm{A}_{1} / \mathrm{A}$ becomes 0.6 near $e=20^{\circ}$. If $S$ is omitted, $A_{1} / A_{\text {e equals }} \mathrm{A}^{\prime} / \mathrm{A}$ at $e=34^{\circ}$ approximately. This value of e changes to about $45^{\circ}$ by the presence of $\zeta$.

To calculate the actual value of the initial stress, the first equation of (2.10) may be used, which is of the form

$$
\frac{{ }^{A}}{A}=f(\Delta, p, q, \delta, \zeta),
$$

where $p, q$ and $\delta$ are supposed to be known quantities. The angular distance $\Delta$ between the epicentre and station for a surface focus is given by

$$
\cos \mathrm{e}=\frac{\alpha}{\mathrm{R}} \frac{\mathrm{dT}}{\mathrm{d} \Delta},
$$

where $R$ is the radius of the core $(3470 \mathrm{Km})$ and $\frac{\mathrm{dT}}{\mathrm{d} \Delta}$ has been computed from the travel times of PCP (Bullen, [6]).

Hence a careful measurements of $A_{1} / A$ will lead to the computation of 5 . ACKNOWLEDGEMENT. The authors wish to offer their sincere thanks to Professor Markus Bảth, Seismological Institute, Uppsala, SWEDEN for suggesting the problem.

\section{REFERENCES}

[1] Dana, S.H. The Amplitudes of Seismic Waves Reflected and Refracted of the Earth's Central Core, Bull. Seism. Soc. Am. 35, (1945), 27-35. 
[2] Ibrahim, A.K. Effects of a Rigid Core on the Reflection and Transmission Coefficients from a Multi-Layered Core-Mantle Boundary, Pure and Applied Geophysics, 91, (1971), 95-113.

[3] Jeffreys, H. The Earth, Cambridge University Press, 1959.

[4] Dey, S. and Addy, S.K. Reflection of Plane Waves Under Initial Stress at a Free Surface, Int. J. Non-1inear Mech. 12, (1977), 371-381.

[5] Biot, M.A. Mechanics of Incremental Deformations, John Wiley, New York, 1965.

[6] Bullen, K.E. An Int roduction to the Theory of Seismology, Cambridge University Press, 1963. 


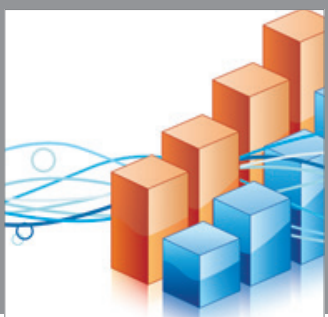

Advances in

Operations Research

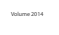

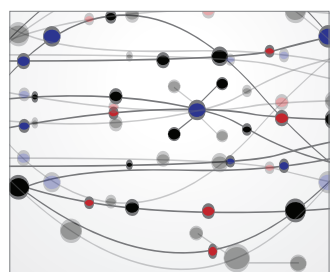

\section{The Scientific} World Journal
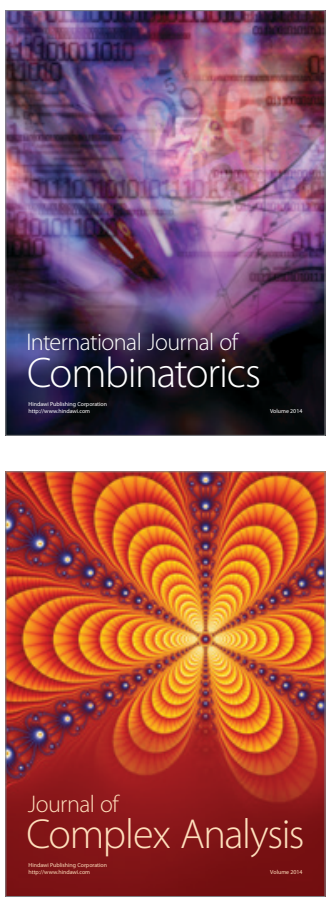

International Journal of

Mathematics and

Mathematical

Sciences
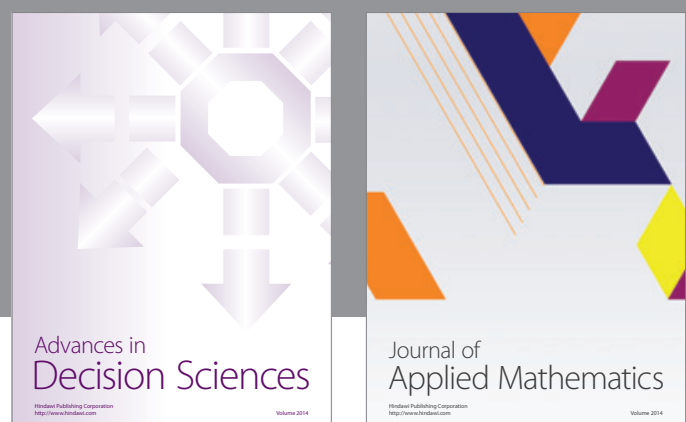

Journal of

Applied Mathematics
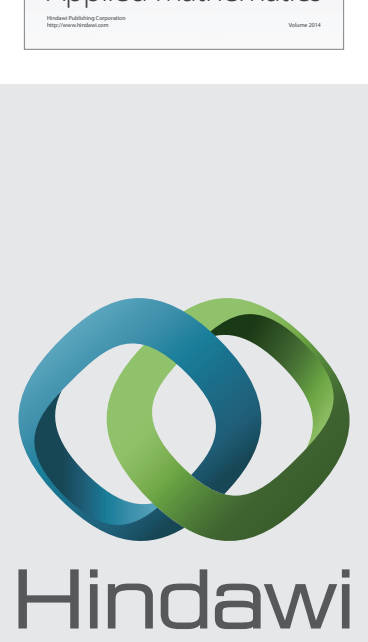

Submit your manuscripts at http://www.hindawi.com
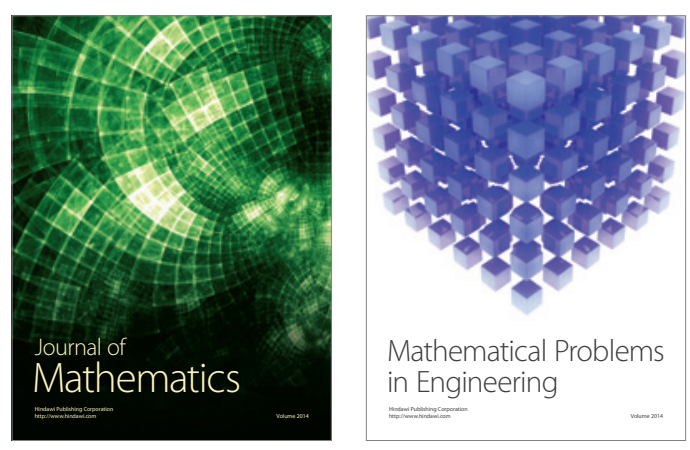

Mathematical Problems in Engineering
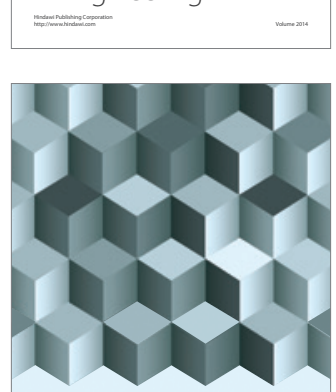

Journal of

Function Spaces
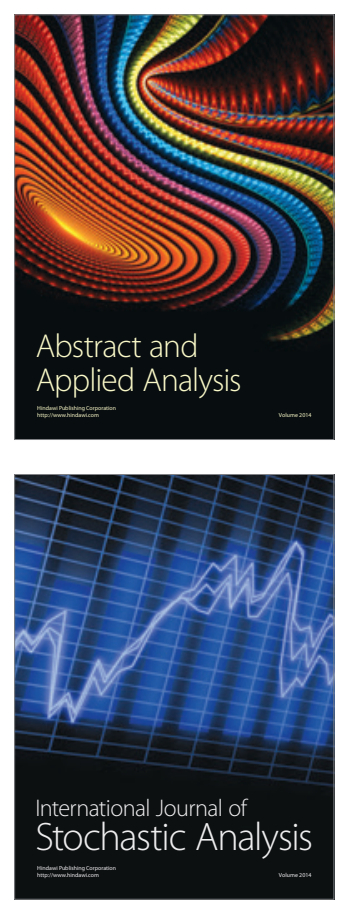

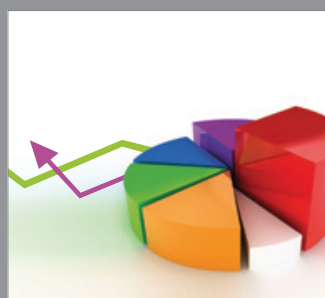

ournal of

Probability and Statistics

Promensencen
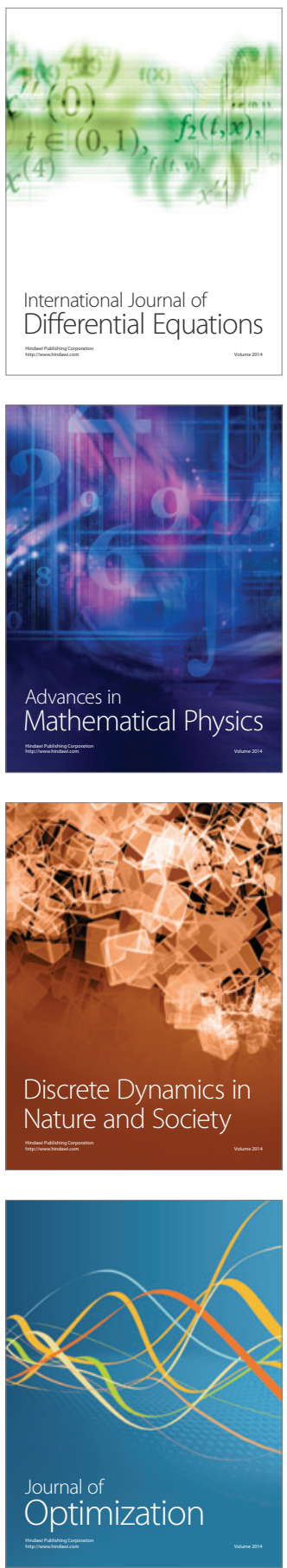